\title{
SWEETs, transporters for intra- and intercellular sugar translocation
}

Joon-Seob Eom ${ }^{1}$, Li-Qing Chen ${ }^{1}$, Davide Sosso ${ }^{1}$, Benjamin T. Julius ${ }^{3}$, I W Lin ${ }^{1,2}$, XiaoQing $\mathrm{Qu}^{1}$, David M. Braun ${ }^{3}$, and Wolf B Frommer ${ }^{1,2}$

\section{Addresses}

1 Carnegie Science, Department of Plant Biology, 260 Panama St., Stanford CA 94305, USA

2 Biology Department, Stanford University, Stanford CA 94305, USA

3 Division of Biological Sciences, Interdisciplinary Plant Group, and the Missouri Maize Center, University of Missouri, 110 Tucker Hall, Columbia, MO 65211, USA

Corresponding authors:

Frommer, Wolf B (wfrommer@carnegiescience.edu)

Key words: glucose, fructose, hexose, sucrose, transporter 


\section{Introduction}

Historically, humans relied primarily on bio-based economies. However, during the industrial revolution a shift occurred towards a fossil fuel economy, most notably in energy, as well as industrial scale production of synthetic chemicals and materials. There is an ongoing discussion regarding a return to an industrial scale bio-based economy. However, such concepts, while at first sight potential innovative drivers of the economy, with the added value of apparent sustainability, also bear major risks. Feeding a world population of 6-9 billion people is already a challenge and has major ecological impacts, thus if industry begins to compete for land and crop production, humans will need new ways to ensure efficient food production and secure biodiversity. In that sense, biofuels were a test case - probably for complex reasons, including the not yet fully developed science, only a major success in Brazil. Yet it initiated a new thinking - the production of high value products from photosynthesis to support the development of other bio-based products - that is returns on high value products could generate enough income to make biofuels competitive and to ultimately transition from the fossil fuel to a bio-based economy. Since today's agriculture is not sustainable, and since productivity is already limiting, scientists and breeders need to find creative new ways to massively increase productivity. Potential targets for crop yield engineering have been carefully explored $\left[1^{\bullet \bullet}\right]$. There is no doubt that carbon allocation in plants must be a central player. Yet this fundamental process is still poorly understood; therefore rational engineering is not yet feasible and demands further research.

Sucrose is produced in photosynthetically active tissues of the leaf and stem, is actively loaded into the phloem, from which it is withdrawn at the various tissues and cells that depend on external sugar supply. At the same time, concentration of sucrose as the dominant osmoticum in the phloem sap, is thought to create the driving force for translocation of all other solutes in the phloem. A bit more than 20 years ago, the first sucrose transporter gene responsible for phloem loading was identified [2], a significant step towards the understanding of sugar translocation. More recently, the lessons learned from model species are starting to be reconciled and implemented in crops such as corn [3]. Yet the story is incomplete: sucrose transporters (SUTs) function as sucrose/proton symporters, thus under standard conditions (high negative membrane potential and 
acidified cell wall), SUTs primarily import sucrose from the cell wall into the cells responsible for long distance translocation. However, sucrose is synthesized from photosynthetically derived precursors in mesophyll cells. Thus at some point sucrose must be released into the cell wall, either directly from mesophyll or from bundle sheath cells, or if sucrose can move from cell to cell via the conspicuous plasmodesmata, release would be expected to occur from cells inside the phloem, such as the phloem parenchyma. Similarly, the sugar efflux transporters for nectar secretion, efflux from seed coat, tapetum, and transmitting tract, the transporters supplying symbiotic and pathogenic microorganisms and the effluxers from the root epidermis, are missing. A unique new family of sugar transporters, the SWEETs, could fill this gap at many of the expected sites. This review focuses on SWEETs from Arabidopsis since the physiological function of these transporters has been studied in most detail in this reference organism, and we highlight a few other SWEETs, e.g. from rice and cassava in the context of their role in pathogen susceptibility.

\section{Identification of SWEETs}

SWEETs were identified by coexpression of membrane proteins with no assigned function with genetically-encoded fluorescent sugar sensors in human HEK293T cells. HEK293T cells are characterized by low endogenous glucose uptake activity and do not show significant sucrose uptake [4-6]. With the help of a FRET glucose sensor we performed a screen for glucose transporters at neutral $\mathrm{pH}$, expecting that this would enable us to identify potential $\mathrm{pH}$-independent uniporters. In this way, we succeeded in identifying members (Clade I and II) of the SWEET family, which contain 17 members that fall into four phylogenetic clades in Arabidopsis (Figure 1 and Interactive Phylogenetic Tree online)[5]. We used an analogous approach to identify novel sucrose transporters, that is, we expressed a FRET sucrose sensor in HEK293T cells and confirmed the absence of sucrose uptake in the human cells. We then performed a screen of unknown membrane proteins including SWEET homologs, and to our surprise found that clade III SWEETs (SWEET9-15) are able to transport sucrose $[7,8]$. We note that, although SWEET do not appear to show strong $\mathrm{pH}$-dependencies, and although they can function in both uptake and efflux assays, formally a uniport function has not been demonstrated. 
Based on the available sequenced genomes annotations (14 April 2015), within Angiosperms, we observed that the number of SWEETs can vary from as low as eight in Amborella trichopoda, the earliest diverging Angiosperm, which is not a member of the monocots or dicots, to as high as 47 in Eucalyptus grandis, a dicotyledonous tree. Intriguingly, in comparison, the Chlamydomonas reinhardtii genome contains five SWEETs, and the Physcomitrella patens genome contains six SWEETs, indicating that there has been a great expansion in SWEET genes in both monocot and dicot lineages [5]. Membership of a SWEET in a clade does not seem to predict the physiological process the protein is involved in, for example, AtSWEET5, AtSWEET8 and AtSWEET13 are vital for pollen nutrition, yet they are found in either clades II or III. Of the SWEETs that have been studied so far, placement of a SWEET in a clade appears to correlate well with the relative selectivity towards mono- versus di-saccharides (Clade I and II prefer hexoses, clade III are efficient sucrose transporters). As stated earlier, all of the clade III SWEETs in Arabidopsis transport sucrose. Also, both clade IV SWEETs in Arabidopsis (AtSWEET16-17) are located on the tonoplast membrane and likely transport fructose. We propose that SWEET genes identified in the future be named according to their phylogenetic relationships to the founding members of the family in Arabidopsis and rice [5], i.e., SWEETs 1-3 in clade I, SWEETs 4-8 in clade II, SWEETs 9-15 in Clade III, and SWEETs 16-17 in clade IV. If due to genome duplication there are multiple genes that are co-orthologous to the closest Arabidopsis or rice gene, we suggest a letter designation follow the gene name to distinguish closely related genes, e.g., Eucalyptus EgSWEET15a-f, rather than each gene being numbered sequentially.

\section{SWEET structure}

SWEETs are different from all other known sugar transporters in that they are heptahelical proteins composed of a tandem repeat of three transmembrane domains (TMs), connected by a linker-inversion TM [5]. Typically, angiosperm genomes contain 15-25 SWEET genes, with the exception of some angiosperm genomes which havea much larger gene copy number of $\sim 4$ (Eucalyptus grandis). Eucalyptus is known to have a large proportion of tandem gene duplications. Chlamydomonas and mosses contain fewer SWEET genes ( 5). SWEET homologs have also been found in animal and human genomes. Eukaryotic SWEETs most likely evolved from ancestral prokaryotic homologs 
that are only $~ 100$ amino acids in length and contain only three TMs (SemiSWEETs) [6,9]. Bradyrhizobium BjSemiSWEET1 mediates sucrose transport, whereas LbSemiSWEET mediates glucose transport. Atomic structures for three SemiSWEETs have been obtained in outside open and occluded states [10,11]. Split ubiquitin yeast twohybrid and split green fluorescent protein (GFP) assays indicated that Arabidopsis SWEETs can oligomerize [9]. Transport-deficient mutants of AtSWEET1 were able to suppress the activity of coexpressed wild-type AtSWEET1, potentially indicating that SWEET oligomerization is necessary for function [9]. The atomic structures are highly valuable for studying the transport mechanism as well as the regulation of SWEETs.

\section{Clade III SWEET9 is essential for nectar secretion}

Nectaries are responsible for producing nectar, which serves as an attractant and reward for pollinators. Although the function and composition of nectar are well characterized, the mechanisms driving nectar secretion have not been determined. Pre-dating the discovery of the function of SWEETs, one of the paralogs had been identified as a nectary-specific gene. Nec1 (as the Petunia homolog of SWEET9 was originally named) is expressed in nectaries and its developmental regulation correlates inversely with nectarial starch content, implying its role in sugar secretion in nectaries [12]. Silencing of the clade III SWEET homolog Nec1 from Petunia triggered male sterility, but a nectar phenotype was not reported [13]. Once the SWEETs had been identified, SWEET9 paralogs were identified as nectary-specific in Arabidopsis, Brassica and Nicotiana [8]. Furthermore, SWEET9 was found to function as a sucrose transporter and be essential for nectar production. These data are consistent with a model in which sucrose synthesized in the nectary parenchyma is secreted by SWEET9 into the apoplasm, where sucrose is partially hydrolyzed by cell wall invertase to produce a mixture of sucrose, glucose and fructose. Thus SWEET9 appears to be responsible for secreting sucrose from the nectarial cells (Figure 2A).

\section{Clade III SWEETs in phloem loading}

Phloem loading is a multi-step process: sucrose is produced in mesophyll cells and traffics between cells possibly through plasmodesmata towards the phloem. Since SUTs load the sieve element companion cell complex with sucrose from the cell wall space, 
sucrose exits the symplasm with the help of sucrose transporting SWEETs. SWEET11 and 12 were found to be expressed in leaves, and subject to diurnal/circadian regulation $[7,14]$. Both proteins, as judged from analyses of GUS reporter fusions, were detected in the phloem. AtSWEET11-GFP was found to localize to the plasma membrane of cells inside the phloem, most likely phloem parenchyma [7], restricting sucrose flux to the interface between the phloem parenchyma and the sieve element companion cell complex deep inside the leaf. Analysis of atsweet11;12 double mutants provided strong evidence for a key role in sucrose translocation out of the leaf; in particular the double mutants accumulated starch in leaves, and radiotracer efflux from petioles was reduced [7] (Figure 2B). Interestingly, AtSWEET13, the closest homolog of SWEET11/12, was upregulated in the atsweet11;12 mutant, implying regulatory systems that adjust SWEET transcript levels. Key future questions are whether SWEETs play similar roles in crop plants, how their activities are coordinated with sucrose anabolism and catabolism and SUT transport activity. The apparent restriction of apoplasmic transfer to the interface between phloem parenchyma and the sieve element companion cell complex may indicate that apoplasmic sucrose levels in other parts of the leaf are low. It may thus be necessary to reevaluate the hypothesis that sugars accumulate to millimolar levels in the leaf apoplasm, which is mainly based on apoplasmic wash fluids, a technology that measures solute efflux into sucrose-free medium and averages across the whole leaf. Cell surface-targeted FRET sucrose sensors may be a suitable tool for monitoring sucrose, such as in the apoplasm surrounding epidermal and mesophyll cells [15].

\section{Role of SWEETs during senescence}

Hexoses, in particular galactose, fructose and glucose, accumulate in senescent leaves, and it has been speculated that SWEET members may also function in remobilization of carbohydrate during senescence [16]. The expression of the AtSWEET15 (SAG29) gene is highly induced in leaves during senescence. Arabidopsis plants overexpressing AtSWEET15 showed accelerated senescence and salt hypersensitivity while atsweet15 mutants were less sensitive to high salinity. However, translational fusion with GUS under control of its own promoter indicate that AtSWEET15 protein does not accumulate to high levels during senescence [17]. This apparent difference may be attributable to overall changes in RNA turnover or to post-translational regulation. Similarly, 
overexpression of OsSWEET5, which can transport galactose, in rice causes growth retardation and early senescence [18]. We observed that overexpression of other SWEETs (e.g. AtSWEET11 and 12) had similar phenotypic effects of growth retardation (Chen and Frommer unpublished; Figure 3). The negative impact of SWEET ectopic expression on plant growth may not be surprising; sugar translocation is limited to a subset of cell types (e.g. phloem parenchyma in the leaf) and polarized. Ectopic expression is expected to lead to a loss in the directionality of sugar flux, and to general leakage of sucrose from cells, thereby reducing phloem loading and causing rather nonspecific effects. Therefore, a potential role in reallocation of carbon under conditions of salinity may be indicated, but additional work is required to evaluate the role of SWEETs in leaves for recapturing of carbon during stress or senescence.

\section{Clade III SWEETs in seed filling}

Based on the finding that SWEETs act as cellular efflux transporters in phloem parenchyma and nectaries, we hypothesized that they may also contribute to assimilate transfer from maternal seed coat to developing embryo. GFP fusions show that AtSWEET11, 12 and 15 are expressed in seed coat and endosperm. Analysis of the triple knockout mutant showed a severe delay in embryo development and a wrinkled seed phenotype at maturity due to lower starch and lipid content and a smaller embryo [17] (Figure 2C). These findings are exciting because the identification of SWEETs involved in seed coat efflux opens up the possibility of identifying the mechanisms that coordinate the delivery of sucrose from leaves with the needs of the developing seed. Extensive studies of the regulation of post-phloem unloading into seeds by osmotic gradients and sucrose provide guidance for further analysis [19]. A generalized model of phloem unloading is presented in Figure 4.

\section{SWEETs in pollen nutrition}

Large-scale gene expression analyses indicate that at least four AtSWEETs may play roles in nurturing the gametophyte [20]. AtSWEET5 (VEXI) is expressed in mature, hydrated and germinating pollen and was found specifically in the vegetative cell of pollen grains, which may supply the generative cell with sugars [21]. Mutation of the hexose transporting clade II AtSWEET8 (RPG1) caused male sterility [5,22]. More 
recent work showed that AtSWEET13 (RPG2) also plays a role in pollen nutrition [23]. In rice, OsSWEET5 is also expressed in anthers.

\section{SWEET recruitment by pathogens}

Apparently, sucrose efflux by SWEETs for phloem loading is restricted to an interface of a few microns deep inside the leaf. One would therefore assume that the apoplasmic space (e.g. between epidermal or mesophyll cells) contains only low sugar levels. Consequentially, the large majority of the apoplasmic space in a leaf may not serve as a good environment for rapid growth of pathogens. Thus pathogens may have to find ways to increase apoplasmic sugar levels in their vicinity. One such example is the direct induction of rice SWEETs by TAL effectors (transcriptional activator-like proteins) from Xanthomonas oryzae. Five out of the 21 SWEET genes in the rice genome have been shown to support pathogen growth [24]. This group of SWEETs all belong to clade III (OsSWEET11-15), and almost all were tested and function as sucrose transporters ([7, 25] and $\mathrm{Qu}$, Sosso, Chen \& Frommer, unpublished). Interestingly, two of the SWEETs had been identified as recessive blight resistance loci, named xal3 and xa25; xal3 corresponds to OsSWEET11 [26] and xa25 to its close homolog OsSWEET13 [27]. Furthermore, OsSWEET14 is targeted by various X. oryzae pv. oryzae (Xoo) strains [28]. Interestingly, OsSWEET12 and 15 have not been found to be targets of Xoo pathovars so far, indicating that the survey of strains is not broad enough to identify pathovars that hijack these transporter genes, that the pathogens have difficulties targeting these genes, or that induction of these proteins has negative side effects. One possible explanation why only sucrose transporting SWEETs can support pathogen growth may be that, in contrast to sucrose pools, hexose pools in the cytosol are limiting, therefore activation of a hexose transporting SWEET may be insufficient to sustain growth of the pathogen population. The understanding of these mechanisms will help engineering broadspectrum resistance by genomic editing of the binding sites of the bacterial TAL effectors using either CRISPR/CAs9 or TALEN technologies in rice [29,30].

Recessive resistance in rice involving SWEETs had originally been seen as unique; however, recently SWEETs have been shown to be induced by TAL effectors from the Cassava blight pathogen $X$. axonopodis pv. manihotis [31] - interestingly, two clade III 
SWEETs were also induced (MeSWEET10 and 15). Mutation of the TAL effector responsible for induction of SWEET10 led to resistance. It is noteworthy that in Cassava blight, invertases are also upregulated, enabling the pathogen to acquire hexoses.

The expression of AtSWEETs from clades other than clade III are induced by Pseudomonas syringae pv. tomato strain DC3000, Golovinomyces cichoracearum and/or Botrytis cinerea [5]. Glucose transporter VvSWEET4 from Vitis vinifera is involved in the interaction with $B$. cinerea, and the mutants of the orthologous AtSWEET4 are less susceptible to $B$. cinerea [32], indicating a role in facilitating glucose efflux into cell wall space to enhance fungal growth. Expression of CsSWEET1 from citrus is stimulated in a TAL effector dependent manner upon the infection of X. citri [33]. Notably, the founding member of the SWEET family, MtN3, was identified as a nodulation-induced gene in Medicago truncatula [34]. Further work will be required to determine whether SWEETs play a role in feeding the symbiotic Rhizobium [35]. SWEETs have been found to be induced in many systems: for example the infection of wheat by Puccinia [36]. It is thus tempting to speculate that SWEETs serve as host susceptibility factors for a wide variety of pathogens including oomycetes and fungi as well as different types of symbionts including mycorrhiza and the microbiota in the rhizosphere.

\section{SWEETs and copper transport}

OsSWEET11 was found to interact with two copper transporters homologs of the COPT family [37]. Data from Wang's lab indicated that OsSWEETs are involved in copper transport. Specifically, they observed that the rice copper transporters COPT1 and COPT5 interacted with OsSWEET11, and that coexpression of all three proteins was necessary to restore copper uptake deficiency of a copper transport-deficient yeast mutant (MPY17 [ctr1 $\Delta \mathrm{ctr} 3 \Delta]$ ). However, copper uptake was not directly measured. The growth phenotype mutant is conditional and is not observed in the presence of glucose. An alternative interpretation of the results may be that COPT1 and 5 mediate low level copper uptake while OsSWEET11 mediates uptake of contaminant sugars from the medium (i.e. the agar), thus partially relieving the dependency of mutant growth on copper. At higher copper levels $(20 \mu \mathrm{M})$, COPT1 and 5 complement MPY17 [38], potentially supporting the proposed mechanism. Alternatively, OsSWEET11 might affect 
plasma membrane targeting efficacy, thereby increasing copper transport activity. It will be important to further characterize this phenomenon in planta.

\section{Vacuolar SWEETs}

The world's sugar supply comes from the vacuoles of sugarcane stems and sugar beet taproots. It is thus of critical importance to understand the interplay of metabolism and transport across the tonoplast. While 10 years ago little was known, now a large number of different sugar transporters on the tonoplast have been identified: proton cotransporters, likely for export of sugars (VGT1 and SUT4), and proton antiporters (TMT) and potential uniporters of the ERD-like family [39-41] (Figure 5). Most recently, homologs of the TMTs were identified as responsible for sucrose accumulation in sugarbeet [42]. Interestingly, clade IV SWEETs have been shown to also function as vacuolar hexose transporters [43-45]. Both AtSWEET 16 and 17 localize to the tonoplast, and AtSWEET17 is a key determinant of leaf fructose content. Despite this massive gain in knowledge, it remains a mystery how this complex suite of transporters is coordinated and how it supports transient accumulation of sugars, and what the key differences are between vacuoles that store low and high levels of sugars. An overview over activities and function of the various SWEETs has been published recently [46].

\section{Regulation of SWEETs}

If SWEETs function as uniporters, which has not yet been formally demonstrated, one might speculate that it would be important to tightly regulate their activity to prevent uncontrolled leakage. One could even envisage that their activity is feedback-regulated by their substrates, coordinated with the activity of their partners, the SUTs in the adjacent cells or regulated by turgor pressure [19]. Post-translational regulatory mechanisms are indicated based on the finding that serine residues in the C-terminus of AtSWEET11 were phosphorylated in response to sucrose addition to seedlings grown in liquid culture [47]. However, because the ectopic transcriptional induction by pathogens leads to sugar secretion, and AtSWEET9 overexpression led to an increase in nectar volume, post-transcriptional control systems may not be essential for SWEET activity. The typically very long cytosolic C-terminus of the SWEETs may serve as a docking platform for protein interactions, however different from the AMTs, for which the C- 
terminus is highly conserved and serves as an allosteric trans-activation domain [48]. SWEET C-termini show much less conservation and are characterized by extensive length variability.

\section{SWEETs in the animal kingdom}

Excitingly, SWEETs have also been identified in humans, and we showed that both a Caenorhabditis elegans and the human homolog mediate glucose transport [5]. In $C$. elegans, one of the seven SWEETs, Swt-1, was identified in a screen for mutants that accumulate lipids using Nile red staining, which could be a secondary effect of glucose accumulation [49]. Mutation of the sea squirt homolog CiSWEET1/Ci-RGA led to early developmental defects, underlining the importance of SWEETs in metazoa [50]. In Drosophila, SWEETs had been identified as genes expressed in embryonic salivary glands [51]. The human SWEET1 shows ubiquitous expression with highest expression

in oviduct, epididymis and intestine as well as in $\beta$-cell lines. HsSWEET1 mainly localized to the Golgi of HEK293T cells [5]. Based on the localization and weak glucose efflux activity, we hypothesize that HsSWEET1 may play a role in the vesicular glucose efflux from $\beta$-cells, the intestine and liver cells [5].

\section{Summary and outlook}

The identification of the large SWEET family as sugar transporters has led to the discovery of many elusive transport steps, including nectar secretion, phloem loading and post-phloem unloading as well as novel vacuolar transporters. Moreover, the human homologs likely play important roles in human physiology. The discovery of SWEETs also enabled the identification of bacterial SemiSWEETs. Atomic resolution structures of the SemiSWEETs have provided insights into the transport mechanism and will enable detailed structure-function analyses. Importantly, SWEETs play key roles in pathogen susceptibility and promise new ways to engineer robust broad-spectrum pathogen resistance. Future studies will be directed towards understanding the regulatory networks that integrate SWEET activity with metabolism and other transporters. Genetically encoded fluorescent sugar sensors targeted to various cellular compartments promise to provide insights into the distribution and the fluxes of sugars which in combination with the analysis of sugar transporter will help us better understand the important 
fundamentals of phloem loading and unloading in model and crop species. Understanding and fine tuning the fluxes between cells in leaves and seeds will likely be key for engineering crop yield potential.

\section{Acknowledgements}

Original work in the Frommer lab was made possible by grants from the Division of Chemical Sciences, Geosciences and Biosciences, Office of Basic Energy Sciences at the US Department of Energy (DOE) under grant number DE-FG02-04ER15542, the National Science Foundation (IOS-1258018) and the Bill and Melinda Gates Foundation. Funding in the Braun lab was provided by a grant from the National Science Foundation Plant Genome Research Program (IOS-1025976). 


\section{References and recommended reading}

Papers of particular interest, published within the period of review, have been highlighted as:

- of special interest

$\bullet \bullet$ of outstanding interest

$\bullet$ 1. Zhu XG, Long SP, Ort DR: Improving photosynthetic efficiency for greater yield. Annu Rev Plant Biol 2010, 61:235-261.

Comprehensive review that dissects the critical steps for yield potential and identifies key targets for engineering yield potential of crops.

2. Riesmeier JW, Willmitzer L, Frommer WB: Isolation and characterization of a sucrose carrier cDNA from spinach by functional expression in yeast. $E M B O J$ 1992, 11:4705-4713.

Identification of the first sucrose transporter using suppression cloning in an engineered yeast mutant.

•3. Slewinski TL, Meeley R, Braun DM: Sucrose transporter1 functions in phloem loading in maize leaves. $J$ Exp Bot 2009, 60:881-892.

Demonstration that the SUT sucrose transporter homolog from maize plays a critical role in phloem loading.

4. Takanaga H, Chaudhuri B, Frommer WB: GLUT1 and GLUT9 as major contributors to glucose influx in HepG2 cells identified by a high sensitivity intramolecular FRET glucose sensor. Biochim Biophys Acta 2008, 1778:10911099.

5. Chen LQ, Hou BH, Lalonde S, Takanaga H, Hartung ML, Qu XQ, Guo WJ, Kim JG, Underwood W, Chaudhuri B et al.: Sugar transporters for intercellular exchange and nutrition of pathogens. Nature 2010, 468:527-532. 
FRET sensor-based identification of SWEETs as hexose transporter in Arabidopsis and rice. Model for role of SWEETs in recessive rice blight resistance.

6. Sosso D, Chen LQ, Frommer WB: The SWEET glucoside transporter family. In Encyclopedia of Biophysics. Edited by Roberts G. Springer; 2013:2556-2558.

•๑7. Chen LQ, Qu XQ, Hou BH, Sosso D, Osorio S, Fernie AR, Frommer WB: Sucrose efflux mediated by SWEET proteins as a key step for phloem transport. Science 2012, 335:207-211.

Identification of clade III SWEETs as sucrose transporters, localization of clade III SWEETs to phloem parenchyma (likely)

๑. Lin IW, Sosso D, Chen LQ, Gase K, Kim SG, Kessler D, Klinkenberg PM, Gorder MK, Hou BH, Qu XQ et al.: Nectar secretion requires sucrose phosphate synthases and the sugar transporter SWEET9. Nature 2014, 508:546-549.

Demonstration that SWEET9 (and SPS) is essential for nectar secretion in Asterids and Rosids.

๑. Xuan YH, Hu YB, Chen LQ, Sosso D, Ducat DC, Hou BH, Frommer WB: Functional role of oligomerization for bacterial and plant SWEET sugar transporter family. Proc Natl Acad Sci U S A 2013, 110:E3685-3694.

Identification of bacterial SemiSWEETs and functional characterization. Evidence that SWEETs can oligomerize and that oligomerization is important for function (defective subunits can inhibit functional units).

$\bullet$ 10. Xu Y, Tao Y, Cheung LS, Fan C, Chen L-Q, Xu S, Perry K, Frommer WB, Feng L: Structures of bacterial homologues of SWEET transporters in two distinct conformations. Nature 2014, 515:448-452.

Atomic resolution structures of two SemiSWEETs in different conformational states (outside open and occluded)

•11. Wang J, Yan C, Li Y, Hirata K, Yamamoto M, Yan N, Hu Q: Crystal structure of a bacterial homologue of SWEET transporters. Cell Res 2014, 24:1486-1489. 
Crystallization of a bacterial SemiSWEET homolog demonstrating that SemiSWEETs form dimers of two triple helix bundles.

•12. Ge YX, Angenent GC, Wittich PE, Peters J, Franken J, Busscher M, Zhang LM, Dahlhaus E, Kater MM, Wullems GJ et al.: NEC1, a novel gene, highly expressed in nectary tissue of Petunia hybrida. Plant J 2000, 24:725-734.

Identification of SWEET9 as a nectary-specific gene.

13. Ge YX, Angenent GC, Dahlhaus E, Franken J, Peters J, Wullems GJ, CreemersMolenaar J: Partial silencing of the NEC1 gene results in early opening of anthers in Petunia hybrida. Mol Genet Genomics 2001, 265:414-423.

14. Haydon MJ, Bell LJ, Webb AAR: Interactions between plant circadian clocks and solute transport. $J$ Exp Bot 2011, 62:2333-2348.

15. Okumoto S, Jones A, Frommer WB: Quantitative imaging with fluorescent biosensors. Annu Rev Plant Biol 2012, 63:663-706.

16. Seo PJ, Park JM, Kang SK, Kim SG, Park CM: An Arabidopsis senescenceassociated protein SAG29 regulates cell viability under high salinity. Planta 2011, 233:189-200.

17. Chen LQ, Qu XQ, Sosso D, McFarlane HE, Samuels AL, Frommer WB: The role of sucrose transporting SWEETs in seed filling of Arabidopsis. Plant Cell 2015 Mar 20. pii: tpc.114.134585. [Epub ahead of print]

18. Zhou Y, Liu L, Huang W, Yuan M, Zhou F, Li X, Lin Y: Overexpression of OsSWEET5 in rice causes growth retardation and precocious senescence. PLoS One 2014, 9:e94210.

๑ 19. Patrick JW, Stoddard FL: Physiology of flowering and grain filling in faba bean. Field Crops Res 2010, 115:234-242.

Excellent review summarizing the steps necessary for seed filling. 
20. Bock KW, Honys D, Ward JM, Padmanaban S, Nawrocki EP, Hirschi KD, Twell D, Sze H: Integrating membrane transport with male gametophyte development and function through transcriptomics. Plant Physiol 2006, 140:1151-1168.

21. Engel ML, Holmes-Davis R, McCormick S: Green sperm. Identification of male gamete promoters in Arabidopsis. Plant Physiol 2005, 138:2124-2133.

22. Guan YF, Huang XY, Zhu J, Gao JF, Zhang HX, Yang ZN: RUPTURED POLLEN GRAIN1, a member of the MtN3/saliva gene family, is crucial for exine pattern formation and cell integrity of microspores in Arabidopsis. Plant Physiol 2008, 147:852-863.

23. Sun MX, Huang XY, Yang J, Guan YF, Yang ZN: Arabidopsis RPG1 is important for primexine deposition and functions redundantly with RPG2 for plant fertility at the late reproductive stage. Plant Reprod 2013, 26:83-91.

$\bullet \bullet_{24}$ Streubel J, Pesce C, Hutin M, Koebnik R, Boch J, Szurek B: Five phylogenetically close rice SWEET genes confer TAL effector-mediated susceptibility to Xanthomonas oryzae pv. oryzae. New Phytol 2013, 200:808-819.

Elegant use of synthetic TAL effectors to test the ability of individual SWEET genes to trigger blight susceptibility in rice. Demonstration that any of the rice clade III SWEETs can cause susceptibility.

25. Zhou J, Peng Z, Long J, Sosso D, Liu B, Eom JS, Huang S, Liu S, Cruz CV, Frommer WB, et al. Gene targeting by the TAL effector PthXo2 reveals cryptic resistance gene for bacterial blight of rice. Plant J 2015 doi: 10.1111/tpj.12838. [Epub ahead of print]

$\bullet$ 26. Yang B, Sugio A, White FF: Os8N3 is a host disease-susceptibility gene for bacterial blight of rice. Proc Natl Acad Sci U S A 2006, 103:10503-10508.

Cloning of the OsSWEET11 gene, which underlies the recessive gene-for gene rice blight resistance. 
27. Liu Q, Yuan M, Zhou Y, Li X, Xiao J, Wang S: A paralog of the MtN3/saliva family recessively confers race-specific resistance to Xanthomonas oryzae in rice. Plant Cell Environ 2011 http://dx.doi.org/10.1111/j.1365-3040.2011.02391.x.

•• 28. Antony G, Zhou J, Huang S, Li T, Liu B, White F, Yang B: Rice xa13 recessive resistance to bacterial blight is defeated by induction of the disease susceptibility gene Os-11N3. Plant Cell 2010, 22:3864-3876.

$\bullet \bullet_{29}$. Jiang W, Zhou H, Bi H, Fromm M, Yang B, Weeks DP: Demonstration of CRISPR/Cas9/sgRNA-mediated targeted gene modification in Arabidopsis, tobacco, sorghum and rice. Nucleic Acids Res 2013, 41:e188.

Application of CRISPR technology to create pathogen resistance in stably transformed rice.

•30. Li T, Liu B, Spalding MH, Weeks DP, Yang B: High-efficiency TALEN-based gene editing produces disease-resistant rice. Nat Biotechnol 2012, 30:390-392.

Use of TALENs to mutate the binding site of the rice blight pathogen Xanthomonas oryzae oryzae TAL effectors to create blight resistance.

31. Cohn M, Bart RS, Shybut M, Dahlbeck D, Gomez M, Morbitzer R, Hou B-H, Frommer WB, Lahaye T, Staskawicz BJ: Xanthomonas axonopodis virulence is promoted by a transcription activator-like effector-mediated induction of a SWEET sugar transporter in cassava. Mol Plant Microbe In 2014, 27:1186-1198.

Demonstration that SWEETs are also susceptibility genes in dicots (Cassava blight)

32. Chong J, Piron M-C, Meyer S, Merdinoglu D, Bertsch C, Mestre P: The SWEET family of sugar transporters in grapevine: VvSWEET4 is involved in the interaction with Botrytis cinerea. J Exp Bot 2014, 65:6589-6601.

33. Hu Y, Zhang J, Jia H, Sosso D, Li T, Frommer WB, Yang B, White FF, Wang N, Jones JB: Lateral organ boundaries 1 is a disease susceptibility gene for citrus bacterial canker disease. Proc Natl Acad Sci U S A 2014, 111:E521-529. 
34. Gamas P, Niebel Fde C, Lescure N, Cullimore J: Use of a subtractive hybridization approach to identify new Medicago truncatula genes induced during root nodule development. Mol Plant Microbe In 1996, 9:233-242.

35. Udvardi MK, Yang L-J O, Young S, Day DA: Sugar and amino acid transport across symbiotic membranes from soybean nodules. Mol Plant Microbe In 1990, 3:334-340.

36. Yu X, Wang X, Wang C, Chen X, Qu Z, Han Q, Zhao J, Guo J, Huang L, Kang Z: Wheat defense genes in fungal (Puccinia striiformis) infection. Funct Integr Genomics 2010, 10:227-239.

37. Yuan M, Chu Z, Li X, Xu C, Wang S: The bacterial pathogen Xanthomonas oryzae overcomes rice defenses by regulating host copper redistribution. Plant Cell 2010, 22:3164-3176.

38. Yuan M, Li X, Xiao J, Wang S: Molecular and functional analyses of COPT/Ctrtype copper transporter-like gene family in rice. BMC Plant Biol 2011, 11:69.

39. Poschet G, Hannich B, Raab S, Jungkunz I, Klemens PAW, Krueger S, Wic S, Neuhaus HE, Büttner M: A novel Arabidopsis vacuolar glucose exporter is involved in cellular sugar homeostasis and affects the composition of seed storage compounds. Plant Physiol 2011, 157:1664-1676.

40. Neuhaus HE: Transport of primary metabolites across the plant vacuolar membrane. FEBS Lett 2007, 581:2223-2226.

41. Schulz A, Beyhl D, Marten I, Wormit A, Neuhaus E, Poschet G, Buttner M, Schneider S, Sauer N, Hedrich R: Proton-driven sucrose symport and antiport are provided by the vacuolar transporters SUC4 and TMT1/2. Plant J 2011, 68:129-136.

• 42. Jung B, Ludewig U, Schulz A, Meissner G, Woestefeld N, Flugge UI, Pommerrenig B, Wirsching P, Sauer N, Koch W et al.: Identification of the 
transporter responsible for sucrose accumulation in sugar beet taproots. Nat Plants 2015 http://dx.doi.org/10.1038/nplants.2014.1.

Identification of a TMT homolog, named TST, for tonoplast sucrose transporter, as being key to sucrose accumulation in sugarbeet taproots.

43. Guo W-J, Nagy R, Chen H-Y, Pfrunder S, Yu Y-C, Santelia D, Frommer WB, Martinoia E: SWEET17, a facilitative transporter, mediates fructose transport across the tonoplast of Arabidopsis roots and leaves. Plant Physiol 2014, 164:777-789.

44. Klemens PAW, Patzke K, Deitmer J, Spinner L, Le Hir R, Bellini C, Bedu M, Chardon F, Krapp A, Neuhaus HE: Overexpression of the vacuolar sugar carrier AtSWEET16 modifies germination, growth, and stress tolerance in Arabidopsis. Plant Physiol 2013, 163:1338-1352.

-45. Chardon F, Bedu M, Calenge F, Klemens PA, Spinner L, Clement G, Chietera G, Leran S, Ferrand M, Lacombe B et al.: Leaf fructose content is controlled by the vacuolar transporter SWEET17 in Arabidopsis. Curr Biol 2013, 23:697-702.

Elegant study which identified clade IV SWEETs underlying genetic availability in vacuolar fructose content in leaves.

46. Chen LQ, Cheung LS, Feng L, Tanner W, Frommer WB: Transport of Sugars. Anпu Rev Biochem 2015 Mar 5. [Epub ahead of print]

•47. Niittylae T, Fuglsang AT, Palmgren MG, Frommer WB, Schulze WX: Temporal analysis of sucrose-induced phosphorylation changes in plasma membrane proteins of Arabidopsis. Mol Cell Proteomics 2007, 6:1711-1726.

Phosphoproteomics demonstrate a sucrose-dependent phosphorylation of SWEETs and $\mathrm{H}^{+} /$ATPases

•48. Loqué D, Lalonde S, Looger LL, von Wirén N, Frommer WB: A cytosolic transactivation domain essential for ammonium uptake. Nature 2007, 446:195-198. 
Allosteric regulation of AMT ammonium transporter activity mediated via Cterminal trans-activation, which is regulated through ammonium-triggered phosphorylation.

49. Ashrafi K, Chang FY, Watts JL, Fraser AG, Kamath RS, Ahringer J, Ruvkun G: Genome-wide RNAi analysis of Caenorhabditis elegans fat regulatory genes. Nature 2003, 421:268-272.

50. Hamada M, Wada S, Kobayashi K, Satoh N: $\boldsymbol{C} \boldsymbol{i}$-Rga, a gene encoding an MtN3/saliva family transmembrane protein, is essential for tissue differentiation during embryogenesis of the ascidian Ciona intestinalis. Differentiation 2005, 73:364-376.

51. Artero RD, Terol-Alcayde J, Paricio N, Ring J, Bargues M, Torres A, Perez-Alonso M: saliva, a new Drosophila gene expressed in the embryonic salivary glands with homologues in plants and vertebrates. Mech Dev 1998, 75:159-162.

52. Edgar RC: MUSCLE: multiple sequence alignment with high accuracy and high throughput. Nucl Acid Res 2004, 32: 1792-1797.

53. Castresana J: Selection of conserved blocks from multiple alignments for their use in phylogenetic analysis. Mol Biol Evol 2000, 17: 540-552.

54. Tamura K, Stecher G, Peterson D, Filipski A. Kumar S: MEGA6: Molecular Evolutionary Genetics Analysis version 6.0. Mol Biol Evol 2013, 30: 2725-2729.

55. Guindon S, Dufayard JF, Lefort V, Anisimova M, Hordijk W, Gascuel O: New algorithms and methods to estimate maximum-likelihood phylogenies: assessing the performance of PhyML 3.0. Systematic Biol 2010, 59: 307-321. 


\section{Legends to Figures}

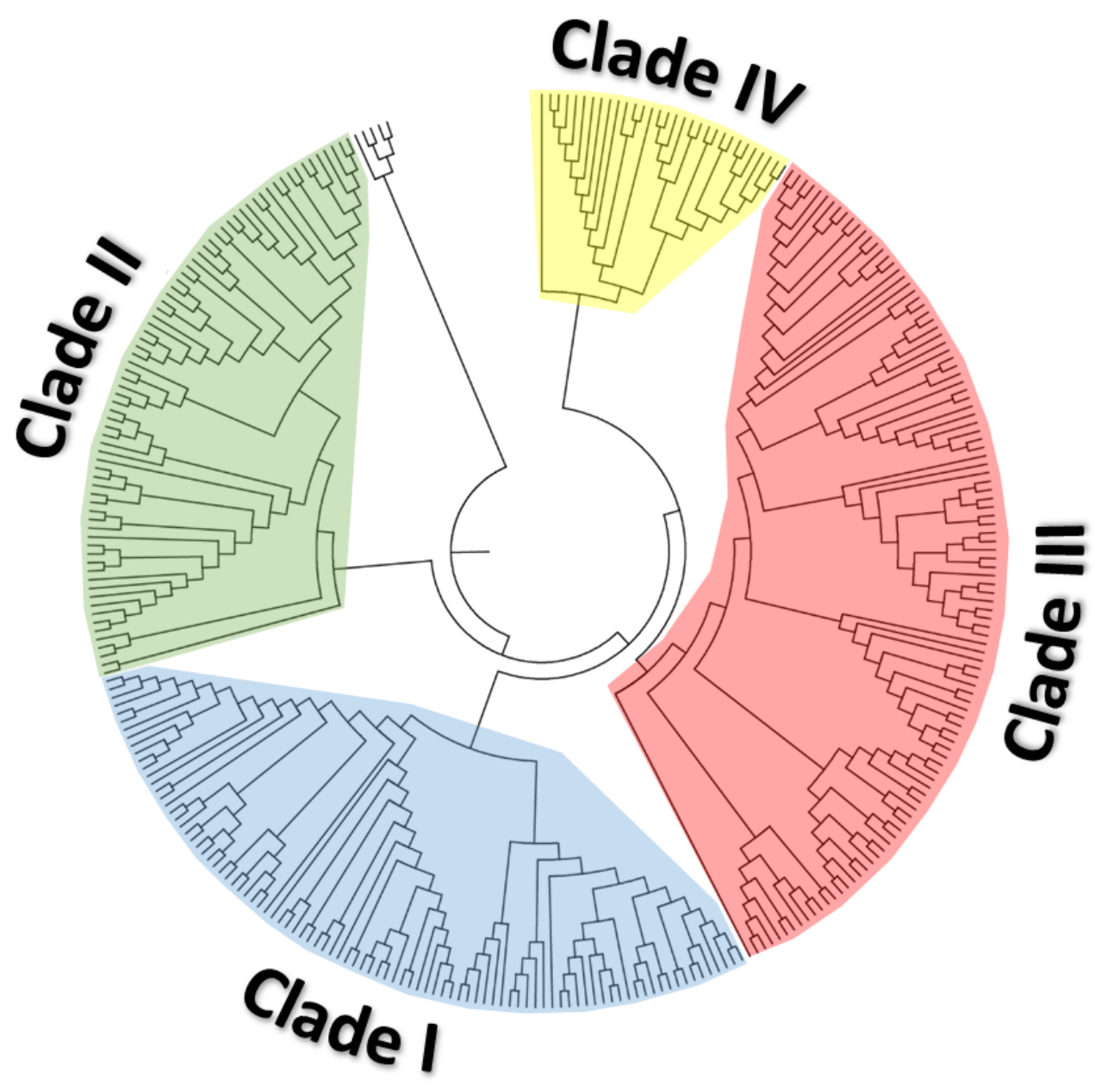

\section{Figure 1}

A phylogenetic tree of SWEET proteins from fifteen plant taxa focusing on the Angiosperms. Accessions were identified through BLAST on Phytozome, Ensembl Plants, NCBI, and CoGe. The SWEET lists were filtered to only include proteins containing two triple-helix-bundle repeats (MtN3 domains). An alignment was generated using default parameters on MUSCLE, and was then curated using Gblocks to exclude non-informative sites $[52,53]$. The maximum likelihood method PhyML was used to generate the phylogeny $[54,55]$. CrSWEET 1 from Chlamydomonas reinhardtii was used as an outgroup. See the Supplementary Figure 1 and the Interactive Phylogenetic Tree online for individual protein names and bootstrap values. 

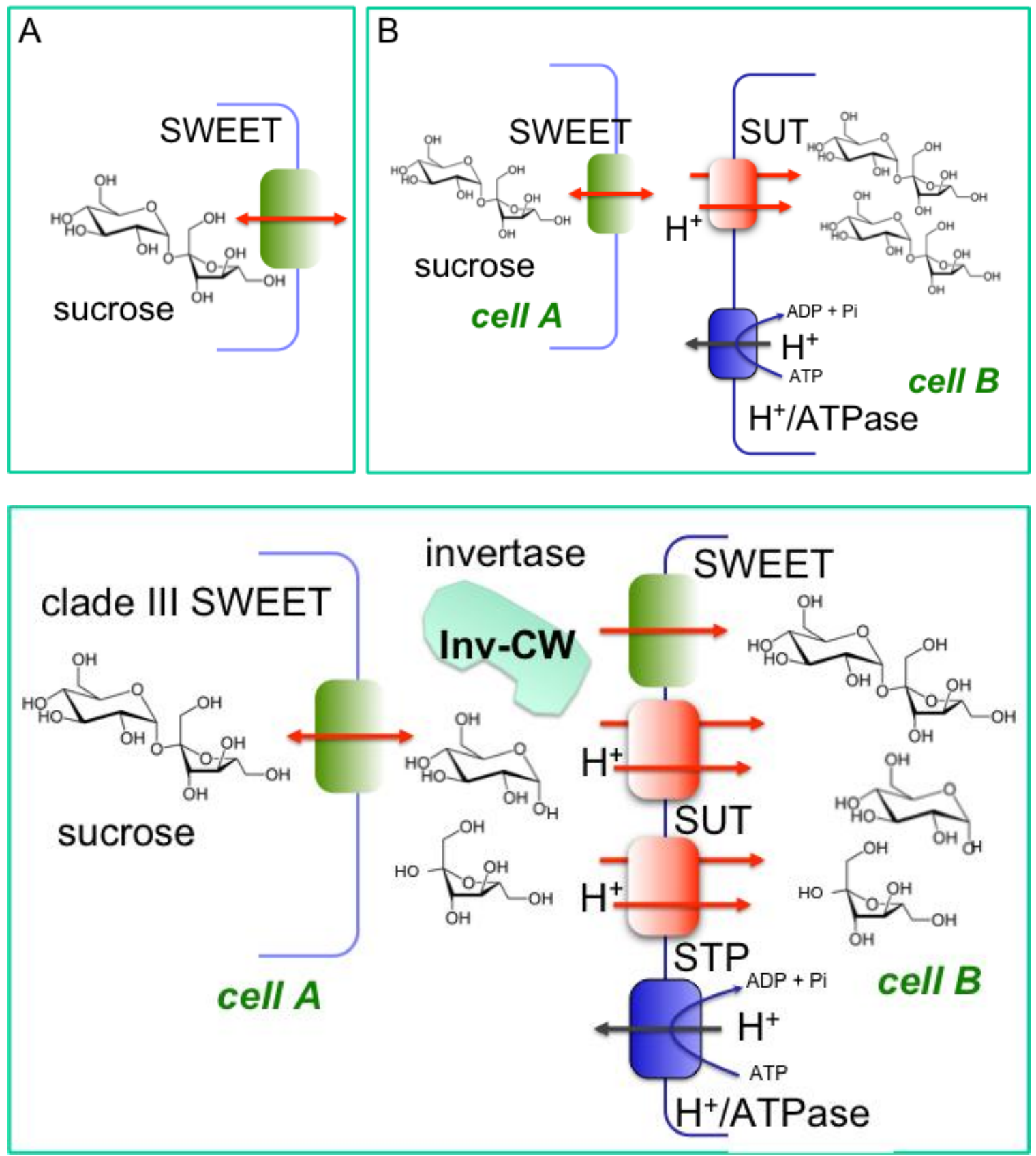

\section{Figure 2}

SWEETs in sugar secretion. (A) Sugars can be exported from cells, for example in the nectary, by SWEETs. (B) SWEETs for the secretion of sucrose from the phloem parenchyma for phloem loading. Cell A secretes sucrose, which is subsequently loaded (against a concentration gradient) into the sieve element companion cell complex (cell B) via a sucrose $/ \mathrm{H}^{+}$symporter SUT. Secondary active sucrose uptake is energized by the $\mathrm{H}^{+} /$ATPase. (C) SWEETs in secretion of sucrose from the seed coat to supply the 
developing endosperm and the embryo. Sucrose secreted from cell A is either taken up by SUTs or SWEETs into cell B, or after cleavage by a cell wall invertase (Inv-CW) taken up via clade I/II SWEETs or by monosaccharide transporters, e.g. of the STP family of hexose $/ \mathrm{H}^{+}$symporters. Note that formal uniport, as indicated by the arrows has not been formally demonstrated for the SWEETs. 


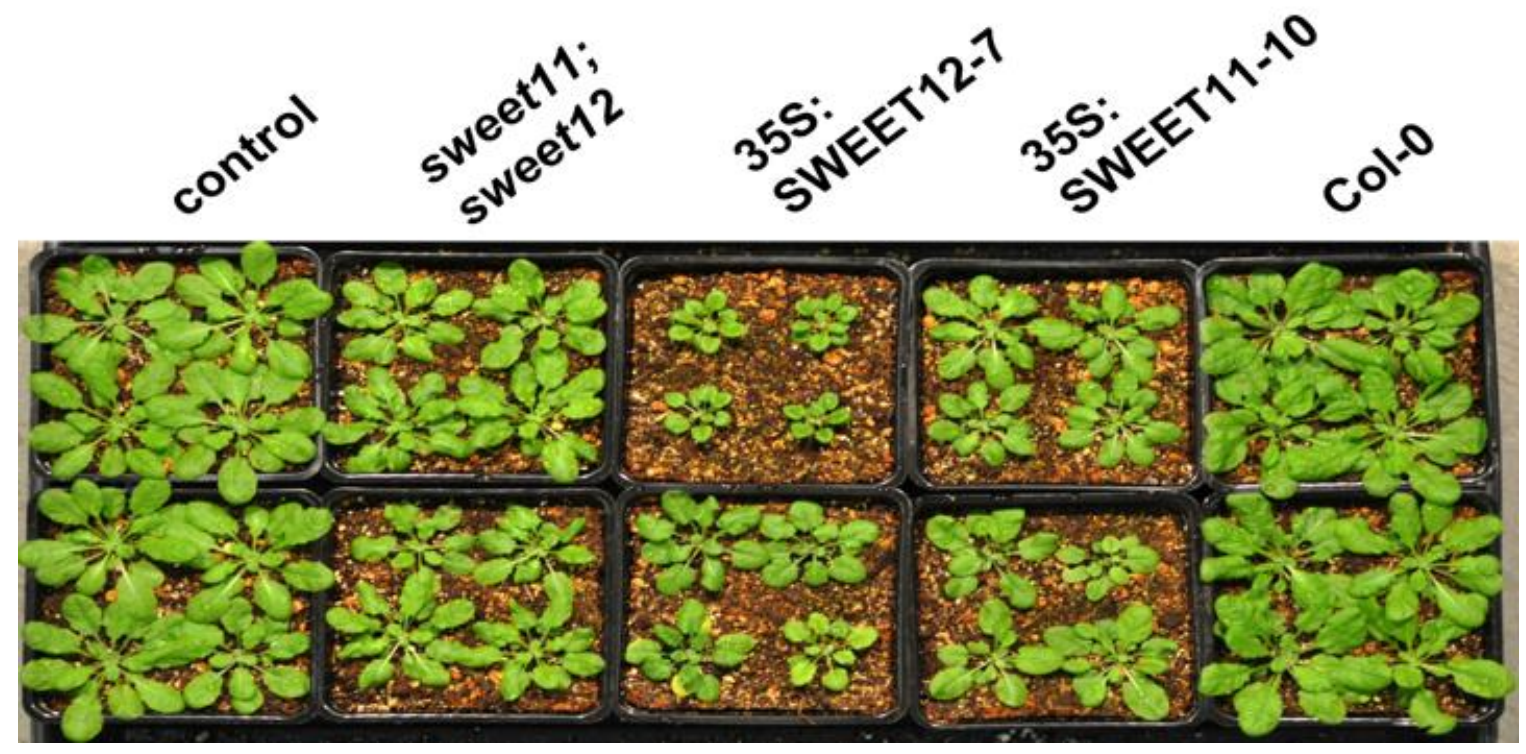

\section{Figure 3}

Negative impact of mutation of ectopic expression of clade III SWEETs. Both the sweet11;12 double mutant [7] and overexpressor lines for SWEET11 and SWEET12 driven by the CaMV 35S promoter (Chen and Frommer, unpublished) show reduced growth. 


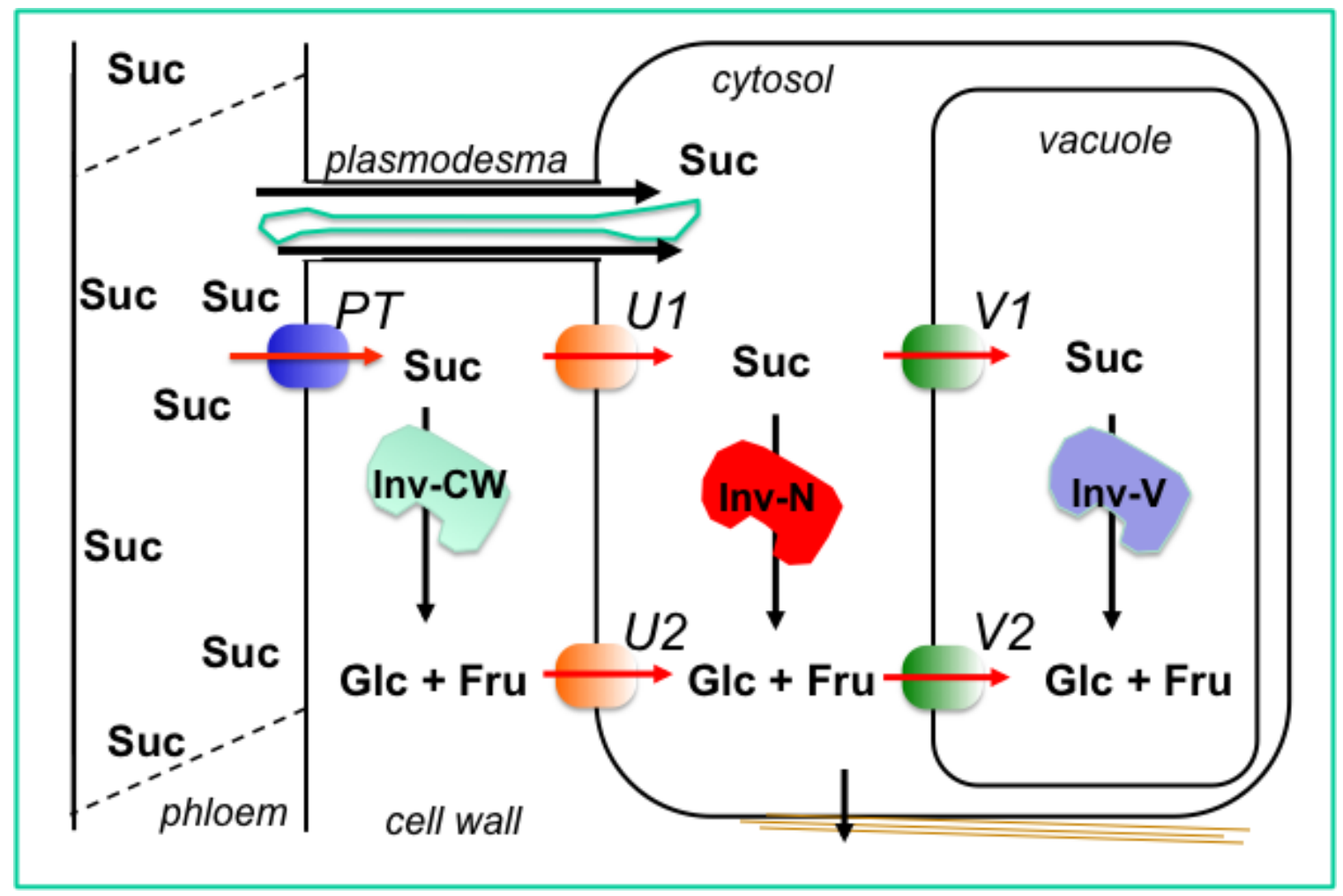

Figure 4

Generic model for principal transport steps and pathways for phloem unloading in various tissues. Sucrose arriving at the phloem endings is either moving symplasmically via plasmodesmata or unloaded (from phloem or after moving symplasmically from postphloem cells) via a transporter (PT); it can be taken up by sink cells using either a sucrose importer (U1), or by a hexose importer (U2) after cleavage by an extracellular invertase (Inv-CW). Imported sucrose can be catabolized to hexoses by a cytosolic invertase (InvN) or sucrose synthetase. Subsequently sucrose or hexoses can be transferred to the vacuole by sucrose or hexose transporters on the tonoplast (V1, V2). The model represents a simplification of the phloem and postphloem unloading pathways. 


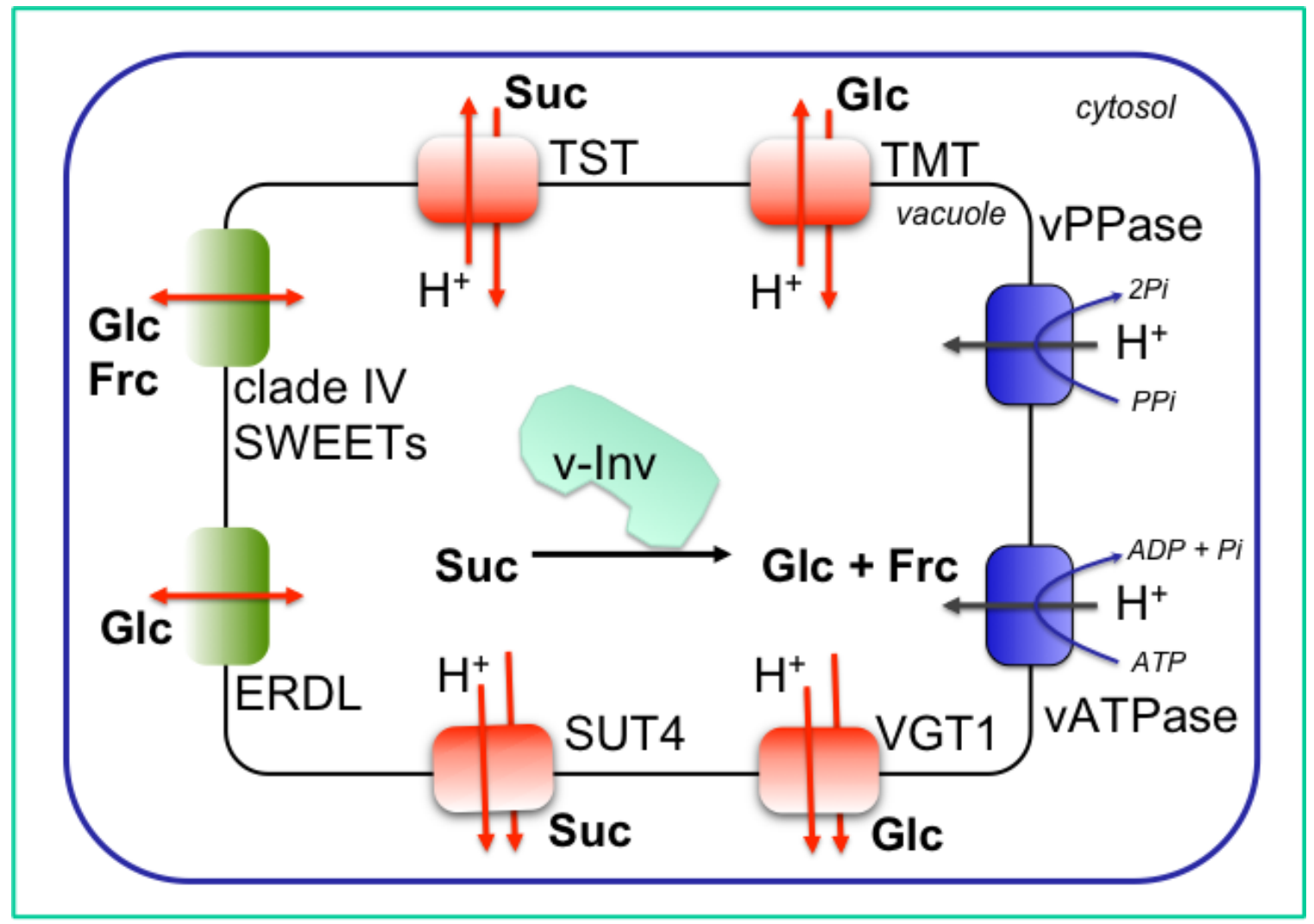

Figure 5

Transporters for vacuolar uptake and efflux of hexoses and sucrose. Three types of sugar transporters have been identified on the tonoplast: proton symporters (SUT4 for sucrose and VGT1 for hexoses), proton antiporters (TMT for monosaccharides and TST for sucrose) and potential uniporters (ERD-like transporters and SWEETs) for hexoses. 